\title{
Research on New Models of Network Travel
}

\author{
Bin Xue, Wei Li, Hongyan Song \\ Hengshui University, Hbei, Hengshui053000 \\ hunter2011@foxmail.com
}

Keywords: E-commerce, Travel, Network travel.

\begin{abstract}
With rapid development and popularity of internet across the world, information technology has been applied to social life and production of people, and enters the fields of traditional business including tourism at an unprecedented rate. The network travel in future should provide a full range of tourism service, which needs to integrate the existing tourism resources to form a platform that tourists can choose the travel process. The paper proposes a new model of e-commerce development in tourism industry--- network travel. Network travel means that tourists communicate with tourism website on internet, arrange tourist routes online, and proposes the required traffic modes and accommodations. Then, the websites arrange the specific journey according to the requirements of consumer. Throughout the journey, the tourists are not accompanied by traditional tour guides but communicate with websites on internet, which can make the tourists enjoy various services provided by websites.
\end{abstract}

\section{Introduction}

Network travel is the development trend of tourism. The emergence and development of network travel will change the present operation mode and system form of travel agency, which not only decomposes traditional hotel functions, but also makes traditional tourism management have revolutionary change.

Network travel has the advantages of large amount of information, rapid update, great independence and timely response. Reservation on internet not only makes the tourists book cheap tickets and hotels, but also can enjoy discounts for recreation and cater.

However, the existing network travel models in our country mainly provide service of booking tickets and hotels for tourists, which is only the upgrade of and is not the real network travel. The network travel in future should provide a full range of tourism service, which needs to integrate the existing tourism resources to form a platform that tourists can choose the travel process. For traditional tourism enterprises, the transformation of large-scale e-commerce technology and powerful tourism resources make them still occupy a position in tourism market. Therefore, only fully mobilizing initiative and accelerating the integration of traditional tourism and network travel can really usher in the spring of tourism market in China.

\section{Status and Tendency of Network Tourism Market Size}

Tourism industry is the information-dependent industry. As the mainstream media in future, the outstanding capability of the next-generation network for audio, video stream and wireless access will become the best choice of tourist destinations and enterprises for promotion. And tourism publicity medium platform, e-commerce platform and service platform will realize integration.

The popularity of remote video conference, telemedicine and distance education makes people break away from the restriction of fixed living space. Traditional independent network_—fixation and motion, and voice and data begins integrating, and a unified network forms, which allows mobile office. And the people traveling outside even can talk with the families with information appliance of IP address. In the next decade, most life of people can spend leisurely, and the leisure tourism industry will be more prosperous.

In the near future, tourism companies can monitor the track of tourist groups at any time. Tourist automobiles transmit the data of driving situations to the relevant departments by using installed 
sensor for vehicle scheduling and maintenance. Mobile network can report the track of mountaineering and exploration tourists in real time. The rescue workers can rapidly locate the tourists encountering danger. Before the rescue workers arrive, internet can make remoter guardianship on them by measuring watches of blood pressure and pulse.

The network travel in future should provide a full range of tourism service, which needs to integrate the existing tourism resources to form a platform that tourists can choose the travel process. Therefore, only fully mobilizing initiative and accelerating the integration of traditional tourism and network travel can really usher in the spring of tourism market in China.

\section{Features of Internetwork Travel}

Development tendency of network tourism. Network tourism is the distribution channel of tourism resources in tourism industry chain. From the perspective of the life cycle process of an industry, the present domestic network tourism has enter the development period with rapid growth, and will develop rapidly in 2-3 years. Ticket and hotel reservation is the basic product and service of network travel service providers because of not involving logistics and payment, and it accounts more than $88 \%$ of the income of service providers. But the market share of packaging service of travel routes facing leisure tourism will reach by $20-30 \%$ in 5 years.

Online reservation service influences and changes the travel habits of consumers, and the convenience and favor promotes network travel consumer group expand rapidly, which forms virtuous cycle.

Competition situation and change of network tourism market. At present, there are 300 distribution channels of network tourism in China which can be divided into three echelons. The first echelon is Ctrip and E xpedia which has monopoly advantage in market. The second echelon is Travel World China and Hengzhong, which has great difference for the first echelon. The third echelon is some small regional service providers. The competition among network travel servers is manifested in resource integration competence and market capability, and the market concentration degree is high now. The network tourism is predicted to enter market maturity in 5 years. The increase of market participants and the pursuit of channels for scale effect cause market integration. And the network travel market is full of competition. The service providers lacking of advantage of core competence will be obsoleted, and the market concentration degree rises again.

The network tourism will begin recessing in 2010. The direct selling share of providers of tourism resources increases. And the share of distribution channels of tourism resources reduces in recession.

Opportunities faced by network travel service providers. The country vigorously supports e-commerce policies and regulations. The soft environment of policies and regulations of network tourism is improving, especially the formulation of defining network travel service will promote the operation and management of network tourism to be more standard, and make the competition more ordering.

Tourism has become the pillar industry in our country. Rapid increase of tourism helps network tourism to develop rapidly. Beijing Olympics in 2008, Winter Olympic of University Students in 2009, Shanghai World Expo and Guangzhou Asian Games in 2010 have great pulling effect on tourism economy in China, which is the golden opportunity for network travel.

Network tourism market develops rapidly, individual leisure traveling consumption requirements are various, and the market potential of domestic commercial tourism and leisure tourism is great, and the scale of potential customer groups for network travel increases rapidly. E-commerce macro environment improves gradually in China. And the idea of online payment will be mature in few years. Domestic tourism market presents the situation of various competitions. And there is no absolute industry leaders monopolizing market. 


\section{System Design of New Mode of Network Tourism}

Overall structure model of system. In order to improve active service quality of network tourism websites and improve satisfaction degree of users, the above hierarchical MAS structure is used to establish new traveling e-commerce system model for users with different levels and requirements. The system has three-layer structures, telling layer MAS, listening layer MAS and management layer MAS. After the user submits the requirement and after data acquisition of Interface Agent, three-layer MAS can coordinate. And one or several alternatives are achieved by series of deductions and learning, and are submitted to the user. The functions are expanded to process information interaction of users. And abstract layer can be used as knowledge accumulation, which plays a significant role in rapid reaction to the same requirements.

System structure is shown in Figure

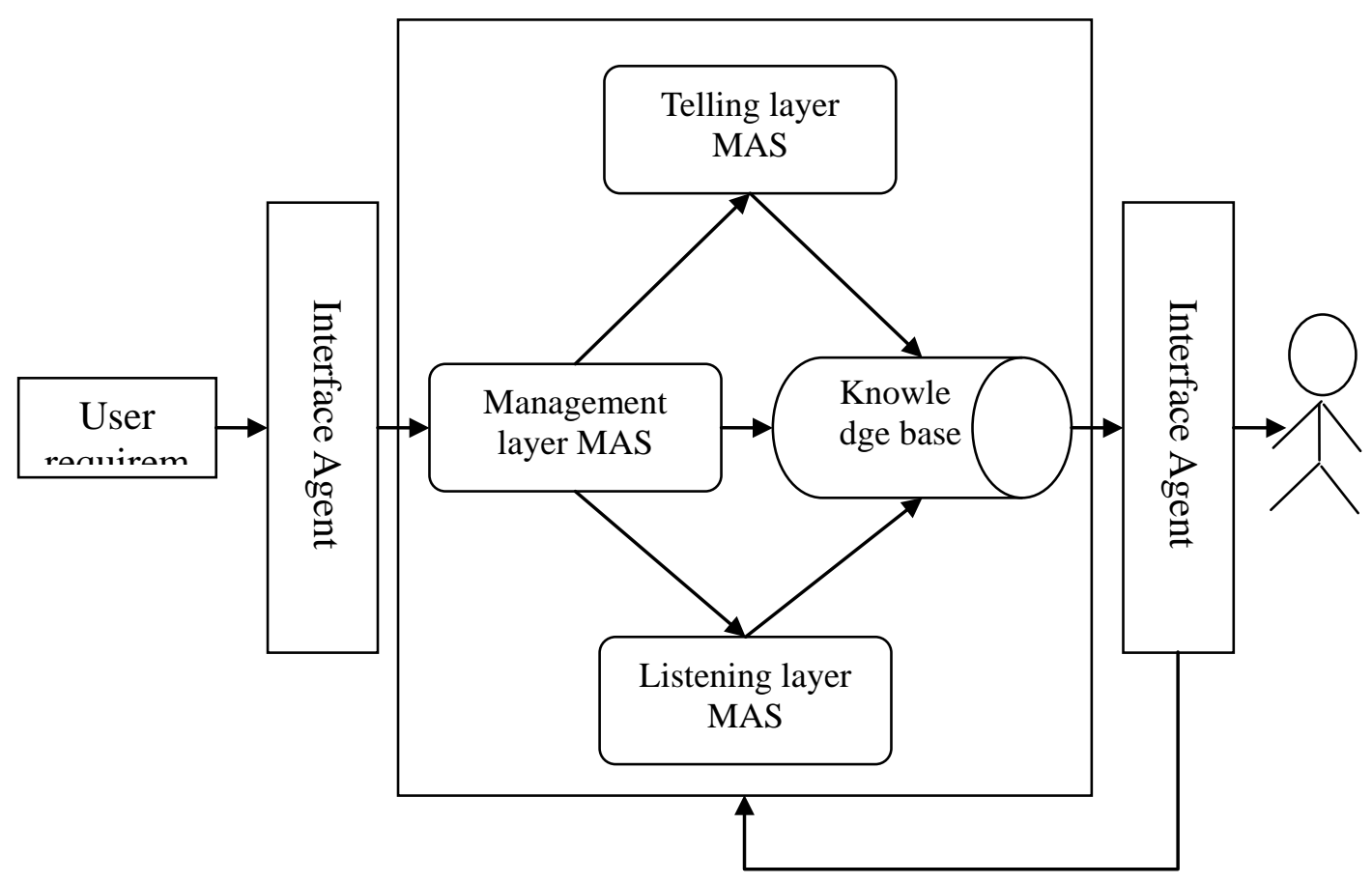

Fig. 1 System structure model

As shown in Figure 1, the system not only has single interface Agent, but also has integrated MAS, which is a composite system. After submitting the requirements, not only the user can achieve a personalized alternative by automatic learning function of the system, but also the setting of user feedback is provided, and the interaction and robustness of the system is enhanced.

In major structure of the system, the telling layer MAS is active. Spider program is used for periodic traversal on the internet, and the cutting-edge information relating to tourism e-commerce is collected, which includes not routes, laws and regulations and fashion ideas. The information is classified based on user preferences. While providing information for the user by message and mail, the resources are stored in the knowledge base of system for reserve.

The management layer MAS takes charge of dispatching local resources. After receiving data of interface Agent, it is firstly analyzed and selected, and the tasks are refined and completed by MAS of other layers. Then, the process results of other-layer MAS are maintained and stored. In the whole process, management layer MAS is in charge of supervision. It can track and draw user state timely, record and reflect thought differences of the user. And the information is stored in learning resources. 
Listening layer MAS is passive. But the layer has the greatest analysis capability and good loyalty. For example, after listening to the requirements, combined with personalized background learning of the user, the optimal scheme is searched in knowledge base of the system. If there is no resource, personalized search engine is immediately started to search on Internet, and the optimal scheme is finally told to the user. And the relevant resources are stored in the knowledge base of the system.

Structure model of Management layer MAS. Management layer MAS plays the role of coordinator in the system. And the structure is shown in Fig. 2.

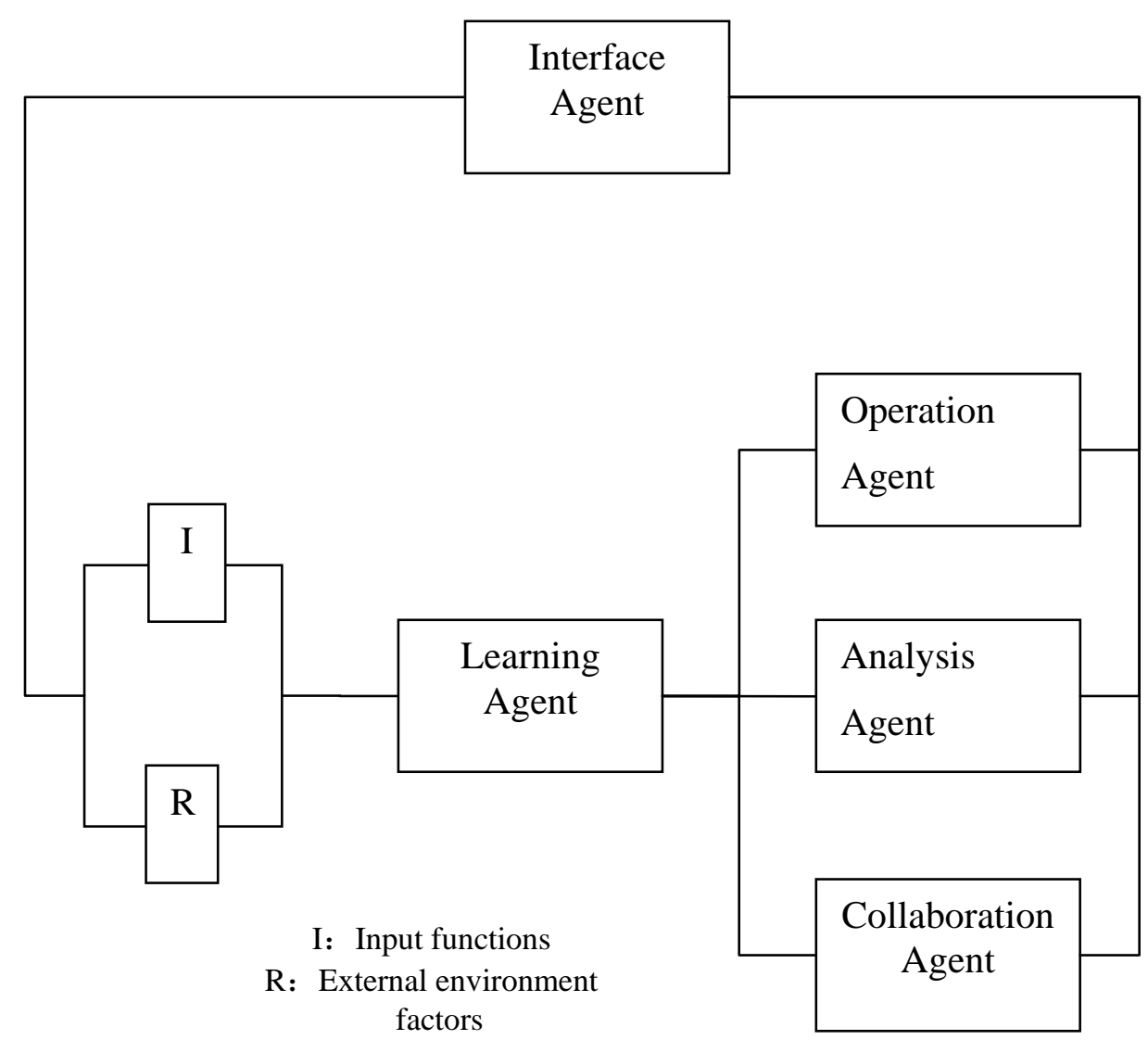

Fig. 2 Management layer MAS model

The structure is based on Internet operation mode. All applications are integrated by Agent, and are published by cross-platform unified protocol with standard language. And the standard user interface is used for display, and it supports many system platforms and database management systems.

Improvement and development. Analyzing the data not only can improve the business process of tourism service enterprises, but also can improve automation degrees, which helps the enterprises to segment the market, redeem the lost customers, retain the present customers and develop new customers, and meet the requirements of customer with low cost and high efficiency. And it can make the service providers improve the satisfaction degree and loyalty of customers, and provider best services.

But the system still needs to be improved. For example, input functions and external environment factors of management layer Agent should be excavated. And the overall model needs code implementation and test run based on establishment.

Introducing Agent technique into tourism e-commerce system model not only overcomes the problems of the system model, but also brings essential reform to the commercial mode, user consumption sense and habits of existing e-commerce enterprises, and has significant influence on 
operation mode of traditional tourism enterprises, which indicates that intruding intelligent technique to traditional industry has great potentials.

\section{Conclusion}

Network tourism is the development tendency of tourism. The emergence and development of network travel will change the present operation mode and system form of travel agency, which not only decomposes traditional hotel functions, but also makes traditional tourism management have revolutionary change. The objective of network travel model is to integrate and expand tourism resources for providing the best tourism service for tourists. In a world, network travel is one of the important requirements for the development of tourism. E-commerce enhances the core competence of tourism enterprises. Therefore, compared with other industries, tourism needs e-commerce, which makes e-commerce easy to be identified in tourism.

\section{Reference}

[1] S.J. Wang, Establishment of network travelling industry chain and analysis on implementation ways, Anhui Agricultural Sciences, 2009,08:3795-3797.

[2] B. Liang, Y. Mao, Research on network travel space in Wuhan, Economic Geography, 2009,07:1214-1217.

[3] S.B. Tian, Chen $\mathrm{Lu}, \mathrm{Wu} \mathrm{Li}$, Prospect on development of network travel combined with virtual reality technology, Market Forum, 2009,09:70-71+65.

[4] P. Zheng, Y.F. Ma, T.S. Li, Research on influencing factors of attitude of university students on network travel information_—_taking Xi'an as an example, Soft Science, 2009,09:47-53.

[5] Y.Y. Guo, Characteristics of information consumption of network travel, Media Today, 2010,02:36-37. 